\title{
Moderation Effect of Neighborhood Walkability during a Physical Activity Intervention in Overweight and Obese Elderly Subjects with Metabolic Syndrome.
}

\section{Antoni Colom Fernández}

Balearic Islands Health Research Institute https://orcid.org/0000-0001-5041-0778

\section{Suzanne Mavoa}

Australian Catholic University

Maurici Ruiz

Universitat de les Illes Balears

Julia Wärnberg

Universidad de Malaga

Josep Muncunill

Balearic Islands Health Research Institute

Jadwiga Konieczna

Servei de Salut de les Illes Balears

Francisco Javier Barón-López

Universidad de Malaga

Montserrat Fitó

Institut Hospital del Mar d'Investigacions Mediques

Jordi Salas-Salvadó

Universitat Rovira i Virgili

Dora Romaguera ( $\nabla$ mariaadoracion.romaguera@ssib.es )

https://orcid.org/0000-0002-5762-8558

\section{Research}

Keywords: Iongitudinal study, physical activity, intervention, walkability index, built environment, older adults, GIS, PREDIMED-Plus trial

Posted Date: December 9th, 2019

DOl: https://doi.org/10.21203/rs.2.18263/v1 
License: (c) (i) This work is licensed under a Creative Commons Attribution 4.0 International License. Read Full License 


\section{Moderation Effect of Neighborhood Walkability \\ 2 during a Physical Activity Intervention in \\ 3 Overweight and Obese Elderly Subjects with \\ 4 Metabolic Syndrome.}

Antoni Colom ${ }^{1,2,}$, Suzanne Mavoa ${ }^{3,4}$, Maurici Ruiz' ${ }^{5}$, Julia Wärnberg, ${ }^{2,6}$, Josep Muncunilli ${ }^{7}$ Jadwiga Konieczna $^{1,2}$, Francisco Javier Barón-López ${ }^{2,8}$, Montserrat Fitón ${ }^{2,9}$, Jordi Salas-Salvadón ${ }^{2,10}$, Dora

7 Romaguera ${ }^{1,2 *}$

${ }^{1}$ Research Group in Cardiovascular Physiopathology and Nutrition Epidemiology (NUTRECOR), Balearic Islands Health Research Institute (IdISBa), University Hospital Son Espases.

$10{ }^{2}$ CIBER Fisiopatología de la Obesidad y Nutrición (CIBEROBN), Instituto de Salud Carlos III.

11 3Mary MacKillop Institute for Health Research, Australian Catholic University, Melbourne, Australia.

$12{ }^{4}$ Melbourne School of Population and Global Health, University of Melbourne, Melbourne, Australia.

13 5Servicio de SIG y Teledetección,Vicerectorat d'Innovació i Transferència, Universitat de les Illes Balears.

14 'Departamento de Enfermería, Facultad de Ciencias de la Salud, Universidad de Málaga - Instituto de 15 Investigación en Biomedicina (IBIMA).

$16{ }^{7}$ Genomics and Bioinformatics Platform', Balearic Islands Health Research Institute (IdISBa), University 17 Hospital Son Espases.

18 'Departamento de Salud Pública, Facultad de Medicina, Universidad de Málaga - Instituto de Investigación en 19 Biomedicina (IBIMA).

20 'Unit of Cardiovascular Risk and Nutrition, Institut Hospital del Mar de Investigaciones Médicas Municipal 21 d'Investigació Mèdica (IMIM).

${ }^{10}$ Rovira i Virgili University, Department of Biochemistry and Biotechnology, Human Nutrition Unit, IISPV, Hospital Universitari Sant Joan de Reus.

Full list of author information is available at the end of the article

*Author of correspondence: Dora Romaguera

Balearic Islands Health Research Institute (IdISBa),

28 University Hospital Son Espases, Bldg I, -1, 07120 Palma, Mallorca, Spain

29 Telf: 871205000 ext 64527; 
33 Background: An infrequently studied question is how the objective measurement of neighborhood walkability modulates physical activity changes during a physical activity intervention program in older adults. We assessed the influence of objectively assessed neighborhood walkability inside the city limits of Palma de Mallorca on the change in physical activity during the intervention program used in the ongoing PREvención con DIeta MEDiterránea (PREDIMED)-Plus trial, a multicenter randomized trial primarily designed to evaluate the long-term impact of an intensive lifestyle intervention on cardiovascular events.

Method: The present study involved 228 PREDIMED-Plus senior participants aged between 55 to 75, recruited in Palma de Mallorca (Spain). Overweight/obese adults with the metabolic syndrome were randomized to an intensive weight-loss lifestyle intervention or a control group. A walkability index was calculated within $1 \mathrm{~km}$ buffers around each participant' baseline home address. Physical activity was assessed using the validated REGICOR Short Physical Activity Questionnaire and accelerometer, at baseline and at 2 follow-up visits (six-months and one-year later). Generalised Additive Mixed Models (GAMMs) were fitted to estimate the association between the neighborhood walkability index and changes in physical activity during follow-up in both the intervention and control group.

49 Results: After one-year of intervention, higher neighborhood walkability (1 z-score increment) was 50 positively and significantly associated with moderate-to-vigorous accelerometer assessed physical 51 activity duration, ( $B=3,44 ; 95 \% \mathrm{CI}=0.52 ; 6.36$ minutes per day). When analyses were stratified by 52 intervention arm, the association was only significant in the intervention group $(B=6.357 ; 95 \% \mathrm{CI}=$ $2.07 ; 10.64$ minutes per day) ( $\mathrm{p}$ for interaction $=0.055$ ). There were no statistically significant associations between neighborhood walkability and self-reported leisure-time physical activity nor 55 brisk walking duration.

Conclusions: The results indicate that the walkability of the neighborhood could support a physical activity intervention, helping to maintain or increase older adults' physical activity.

Keywords: longitudinal study; physical activity; intervention; walkability index; built environment; older adults; GIS; PREDIMED-Plus trial. 
62

63

64

65

66

67

68

69

70

71

72

73

Given the rapid increase of the elderly population $[1,2]$ combined with their rising trend of insufficient physical activity [3, 4], active aging has become a key issue for public health. This makes it necessary to implement effective individual-level physical activity intervention programs promoting physical activity among older adults. Physical activity intervention programs among older adults have been shown to significantly increase physical activity, which has been confirmed in two recent systematic reviews $[5,6]$. However, little is known about whether the environments in which older adults are encouraged to be active play a role.

The built environments are well known to promote active aging at the population level [7]. Older adults spend more time within their immediate neighborhood environment [8]. This situation makes them especially sensitive to physical barriers towards health promotion efforts [9]. Multiple attributes such built environments are frequently conceptualized and assessed using a validated walkability index, that takes into account residential density, intersection density, and land use mix [10]. High residential density within neighborhoods means that more people live in the same area. This, in turn, creates the need for increased goods and services, such as sustainable recreational facilities, public transport, and local businesses, resulting in a wide variety of destinations for local residents. Higher intersection density makes the area more connected, offering more direct and greater number of alternative travel paths between destinations. Land use mix measures the diversity of destinations within the area that are accessible without using a car. In consequence, neighborhoods with high population density, well-connected streets and high numbers of mixed destinations within walking distance, are characterized by a walkability index. Within this context, living a high walkable community is likely to be a key aspect of built environments that promotes physical activity practice [11].

The strategic combination of individual-level physical activity intervention programs with environmental factors that support physical activity could be synergetic to engage older adults in regular physical activity [12]. Socio-ecological models postulate that physical activity behavior might be influenced at multiple levels, (individual, social, and environmental factors) by complex and dynamic interrelations $[13,14]$. Therefore, it is reasonable to expect that during physical activity intervention programs we should observe interrelations between individual, social, and environmental factors on the acquisition of physical activity behaviors.

The PREvención con DIeta MEDiterránea (PREDIMED)-Plus study, an ongoing multicenter randomized trial primary designed to evaluate the long-term impact of an intensive lifestyle intervention on cardiovascular events, recently published the effectiveness of the physical activity intervention component. This secondary analysis showed promising individual-level effectiveness in the increment of physical activity after one-year of a lifestyle intervention program for older overweight/obese participants with metabolic syndrome [15].

In the present study, using a sub-sample of participants from one of the recruitment centers of the PREDIMED-Plus, we evaluated whether older adults assigned to a tailored intervention to increase their physical activity who are exposed to more walkable neighborhoods will better comply with physical activity recommendations compared to those in less walkable neighborhoods. The current study aimed to 1) Assess the association of neighborhood walkability, on one-year change in physical activity assessed by accelerometer and self-reported data and 2) Assess whether this association is strengthened with a physical activity intervention.

\section{Methods}

\subsection{Study Population}

This study was part of the ongoing PREDIMED-Plus study, a multicenter, parallel-group, randomized trial. Details of the trial, measurements, CONSORT flow chart and data collection have been published elsewhere [16] and the protocol detailing intervention activities is fully available at 
http://predimedplus.com. Briefly, the main objective of PREDIMED-Plus was to evaluate the effect

111 of an intensive lifestyle intervention on cardiovascular disease prevention, in overweight and obese

112 participants with the metabolic syndrome. Participants were randomly assigned, in a 1:1 ratio, into

113 two study condition groups. The intervention group was assigned to an intensive weight-loss

114 lifestyle intervention including energy-restricted Mediterranean diet, behavioral support, and

115 individualized physical activity promotion. The control group received unrestricted-energy

116 Mediterranean diet advice and without any advice to increase physical activity. The trial was

117 registered at the International Standard Randomized Controlled Trial

118 http://www.isrctn.com/ISRCTN89898870 and the study protocol was approved by Research Ethics

119 Committees from all 23 recruitment centers.

120 The present analysis was designed to measure neighborhood walkability and its components in

121 a subset of participants from 1 of the 23 recruitment centers (University Hospital Son Espases) of the

122 PREDIMED-Plus trial. This secondary analysis was approved by Committee of Research Ethics of

123 the Balearic Islands (CEI-IB) (number IB-2242/14-PI) and all participants provided written informed 124 consent.

From May 2014 to November 2016, 335 participants were recruited from primary healthcare facilities near the University Hospital Son Espases (HUSE), Balearic Islands, Spain. PREDIMED-Plus participants were eligible if at enrolment they were men aged 55-75 years and women aged 60-75 years who were overweight or obese (body mass index (BMI) $\geq 27$ and $<40 \mathrm{~kg} / \mathrm{m}^{2}$ ) without documented history of cardiovascular disease (CVD) and fulfilled at least three of the metabolic syndrome criteria [17].

131 Data for the present analysis was sourced from the completed PREDIMED-Plus database, dated 13219 Mar 2019. We included 228 participants in the analytic sample, excluding participants who 133 reported living outside the city limits of Palma de Mallorca or had no accelerometer data. Among 134 them, 106 participants were allocated to the intervention group and 122 to the control group (Figure 135 1). This analytic sample did not differ from the total HUSE cohort $(n=335)$ in terms of their main 136 characteristics (sex, age, baseline self-rated health, BMI, educational level, baseline 137 accelerometer-assessed and self-reported moderate-to-vigorous physical activity, self-reported 138 leisure-time brisk walking). The ongoing intervention was planned for 6-years, with a further 139 2-years of extended follow-up for collection of clinical events. For the present analyses, we only used 140 baseline, 6-month and 12-month follow-up data collected between 2014 and 2017.

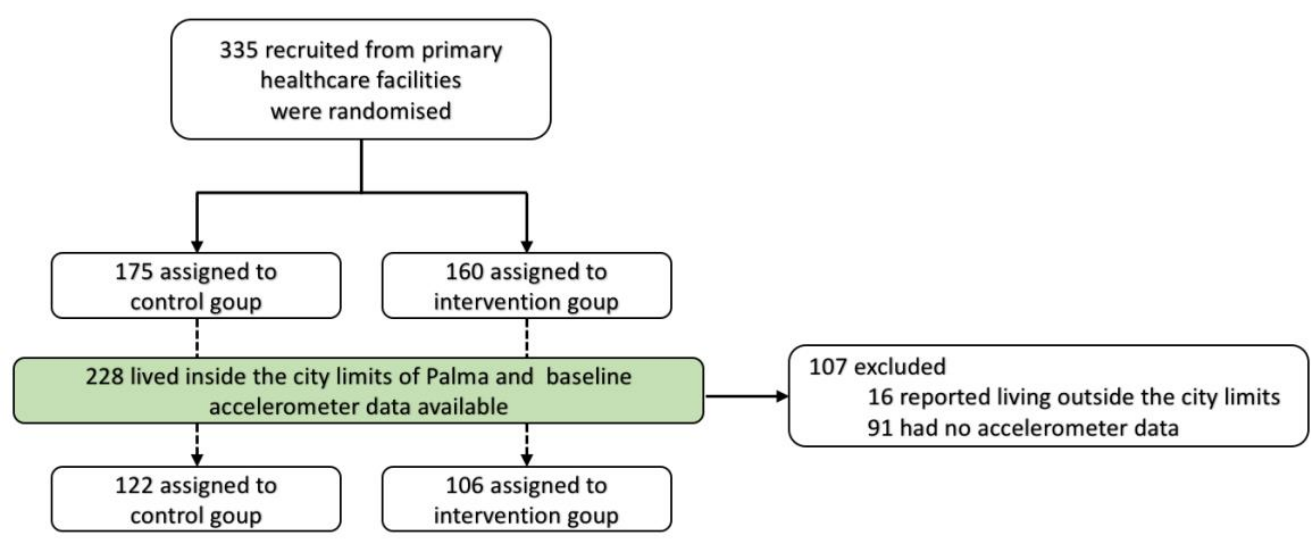

142 Figure 1. Flow chart in this analysis of the PREDIMED-Plus trial. 
During the ongoing PREDIMED-Plus trial, dietitians who received additional training in physical activity, delivered the dietary and physical activity intervention program. In the time period analyzed in the present study (first year of the ongoing trial), participants in the intervention group received a face-to-face educational program. The intervention program included 12 individual one-hour sessions, 12 telephone calls, and 12 one-hour group sessions ( 3 of them devoted to physical activity). The intervention program is founded on the behaviours change theoretical framework, all detailing behaviours change techniques used have been published elsewhere [15]. Throughout the intervention, participants were encouraged to gradually increase their physical activity levels to at least 150 minutes/week of moderate-to-vigorous physical activity (MVPA), with the ultimate goal of walking at least 45 minutes/day, 6 days per week. Feedback, informational materials, motivation, and self-monitoring techniques were used during the follow-up sessions. To self-monitor steps and progressively increase the time spent in brisk walking, a pedometer was provided to each participant (Yamax SW200 Digi-Walker). The degree of adherence to the physical activity intervention was monitored quarterly and problem-solving interviews were carried out to overcome any difficulty.

159 Previous reported results of the PREDIMED-Plus physical activity intervention program showed effective increasing of physical activity after one-year of a physical activity intervention program [15].

\subsection{Neighborhood walkability index}

We objectively measured our exposure, neighborhood walkability, at baseline with multiple geographic scales $(0.5 \mathrm{Km}$ and $1 \mathrm{Km}$ buffers) around participants' residential addresses [18]. The buffers were created following the generally considered the gold standard, sausage buffer methodology described by Forsyth and colleagues [19]. In short, we obtained the centerline data of the street network from the Carto Ciudad project (www.cartociudad.es) dated 11 April 2018. Excluding routes restricted to pedestrians, such as highways, from the network, and applying ArcGIS Service Area function, we selected walkable streets within $0.5 \mathrm{Km}$ and $1 \mathrm{Km}$ of each participant' residence location. Lastly, for each participant' street network, we buffered $30 \mathrm{~m}$ either side of the street centerline.

For each street network buffer, we extracted features of the neighborhood walkability. Using ArcGIS V10.5.1 software (ESRI, Redlands, CA, USA) and following European recommendations [20], we applied the methodology adapted from the work of Frank and colleagues [10] to identify residential density, intersection density and land use mix.

The first measure, residential density, was calculated by counting the units of dwellings (single family homes) per square kilometre of residential land area using data acquired from the 2018 Spain cadastre. The next step was to determine the intersection density by counting the number of three or more legs intersections per square kilometre from the Carto Ciudad. The final component, land use mix, was calculated using five of the eleven categories of the 2018 Spain cadastre: residential (single family homes), commercial (shops, markets and supermarkets), eateries (bars, cafes and restaurants), entertainment (cinemas, theatres and music bars) and cultural (libraries, museums, churches and parish centers). After undertaking a literature review we selected these identified cadastre categories and excluded land classifications that did not fall within these categories (e.g., 
industrial, agricultural, offices, health and charity, singular buildings) to better capture recreational physical activity and walking [21, 22]. Once these were identified, we applied [Equation (1)], a variation of the entropy formula previously developed by Frank et al [23], explained elsewhere [24].

$$
\text { Land use mix }=-1\left(\sum_{i=1}^{n} p_{i} * \ln \left(p_{i}\right)\right) / \ln (n)
$$

Where $p_{i}$ is the proportion of the buffer covered by land use $i$ against the summed area of total land use classes, and $n$ is the number of land use class of interest (in our case 5 ). When $p_{i}$ was $\leq 0$, then $\ln \left(p_{i}\right)$ was replaced by 0 .

From here, we obtained an entropy score between $0-1$, with 0 representing homogeneity of land uses and 1 representing heterogeneity of land uses.

Once each value was calculated (residential density, intersection density, and land use mix), each measure was normalised using a z-score, indicating the standard deviation above the mean of the raw value for each category. Finally, walkability index was calculated by summing the z-scores of residential density, intersection density, and land use mix.

\subsection{Outcome Measure: Physical activity}

We assessed the outcomes of the present study, accelerometer and self-reported assessed physical activity, at baseline, at 6 and at 12 months. At each time point, trained dietitians asked the participants to wear an accelerometer for 7 days and completed with each participant the validated REGICOR Short Physical Activity Questionnaire (RSPAQ) [25].

Accelerometer assessed MVPA duration (minutes/day) (AA-MVPA) was assessed using wrist-worn triaxial accelerometer GENEActiv activity monitors (GENEActiv ActivInsights Ltd, Kimbolton, UK). We asked participants to use the device on the non-dominant wrist for 7 days without removing it during water activities and sleep. The wrist-worn triaxial accelerometer was sampled at 40 hertz $(\mathrm{Hz})$ with $\mathrm{a} \pm 8$ gravity units $(\mathrm{g})$ dynamic range. Accelerometer files were processed using R-package GGIR (version 1.2-5), available on CRAN (https://cran.r-project.org). Following other authors' recommendations [26,27] we defined non-wear day as $\geq 2$ hours of 0 count values per day and a minimum wear time as $\leq 4$ valid days with $\geq 1$ weekend day. Using the older adults cutoff points proposed by Hildebrand and colleagues, we determined time spent in MVPA by summing each minute greater than 100 milligravity [28]. Finally, we estimate the average minutes/day of AA-MVPA at baseline and at 6 and 12 months.

Self-reported leisure-time moderate-to-vigorous physical activity duration (minutes/day) (SRLT-MVPA) was assessed at baseline and each time point follow-up using the validated questionnaire RSPAQ. Frequency, duration, and intensity of 6 types of activities (walking, brisk walking, walking on trails/hiking, gardening, climbing stairs, and sport activities), were recorded. Among Spanish adults in a Mediterranean context, the RSPAQ has shown reasonable validity for measuring moderate and vigorous activities when compared to the accelerometer derived physical activity (Spearman correlation coefficient for moderate intensity 0.40 , and 0.26 for vigorous intensity) [25]. For the purpose of this study we selected MVPA's using the Compendium of Physical Activities [29].

Also, a domain-specific physical activity self-reported leisure-time brisk walking duration (minutes/day) (SRLT-BW) was assessed at baseline and each time point follow-up using RSPAQ' question on days per month and minutes per day of leisure-time brisk walking. 
Dichotomous outcome measures were computed to represent the PREDIMED-Plus physical activity intervention goals. Engaging at least 150 minutes of MVPA per week and engaging at least 45 minutes of brisk walking per day, 6 days per week. This goals are in line World Health Organization recommendations for adults of this age group [30].

\subsection{Covariate Assessment}

Covariable information was collected at baseline in a face-to-face survey from which the following socio-demographic variables were extracted: age, sex, education level (dichotomized as primary school or less or secondary school or higher) and self-rated health (excellent/very good/good vs. fair/poor).

We calculated body mass index (BMI) by dividing weight $(\mathrm{kg})$ by height squared $\left(\mathrm{m}^{2}\right)$. Weight $(\mathrm{kg})$ and height $(\mathrm{m})$ were directly measured at each time point follow-up using a wall-mounted stadiometer and electronic scale, respectively.

To calculate neighborhood deprivation, census section units for participant were identified using the location of each participant' residence, reported at baseline. For each geographic scale buffer, we extracted weighted measures of census deprivation index from 2011 dataset acquired from the Sociedad Española de Epidemiología [31]. This deprivation index has been built for total Spanish State Census sections, combining information from six socioeconomic indicators (percentage of manual working population, percentage of the temporary working population, percentage of the unemployed population, percentage of the population with insufficient education (population that cannot read or write, went to school for less than 5-years, without completing high school), percentage of young population with insufficient education and percentage of main dwellings without Internet access). We categorized the 2011 deprivation index weighted measures into deciles, where 1 is the least disadvantaged geographic scale buffer and 10 is the most disadvantaged.

Rain conditions (period without rain and with rain) for each accelerometer wearing period (baseline, 6 and 12 months) were assessed using the application programming interface provided by the OpenData service of the Agencia Estatal de Meteorología (AEMET). From each weather station closest to the participant' residence we determined the rain conditions.

\subsection{Data analytic plan}

Data were analyzed to assess: 1) the association of neighborhood walkability with one-year change in AA-MVPA, SRLT-MVPA and SRLT-BW; and meeting the PREDIMED-Plus trial physical activity intervention goals and 2) whether this association was strengthened by a physical activity intervention by evaluating the interaction between walkability and study condition (intervention or control group) and conducting stratified analyses.

In order to avoid type I and type II errors, we applied a protocol for data exploration proposed by Zuur et al. [32] Cleveland dotplots were used at all variables to detect outliers and if necessary, raw data was checked for errors and to determine whether the observed values were reasonable. Observations of one participant were omitted during the outliers' analysis. Verification of homogeneity of variance was done plotting residuals vs. fitted values and the assumption of 
normality was tested by plotting histograms of residuals. We tested collinearity calculating the variance inflation factor (VIFs), all covariate VIFs were less than 2. Finally, dependence in the observations of the response variable was checked in the raw data before the analysis.

We computed descriptive statistics (means, standard deviations and percentages) at baseline for all variables and specific study conditions. Continuous normally distributed baseline characteristics were compared using the t-test, continuous non-normally distributed baseline characteristics were compared using the Kruskall-Wallis test and categorical baseline characteristics were compared using chi-squared or exact Fisher test when the expected frequencies are less than 5 in some cell.

In order to investigate how much of a built environment feature leads to healthy behaviours previous researchers recommended consider models that allow to explore non-linear forms [33]. Generalized additive mixed models (GAMMs) can allow for flexible, non-linear forms that are useful for uncovering dose-response, linear, non-linear and threshold relationships and can account for dependency in residuals (multiple measures taken on the same participant sampled from selected neighborhood units) [34]. Taking into consideration the multiple observations on the same participant, we include a dependence structure using random effects. To consider the dependence between built environments features and health behaviours at the same neighborhood socioeconomic status, we included a dependence structure using nested random effects of participants sampled from same neighborhood deprivation index. Therefore, all models accounted for two nested levels of variability in the outcome, variability at the person level (ie, between-participant multiple observations differences) nested at the area-level socioeconomic status (ie, clustering effects at the neighborhood deprivation index level).

We used GAMMs with Gaussian or Binomial variance and identity link function to evaluate the effects of neighborhood walkability on respectively the duration of each physical activity variable or engaging in their recommendations ( $\geq 150$ minutes/week AA-MVPA, SRLT-MVPA and $\geq 45$ minutes/day 6 days per week SRLT-BW). All coefficients were adjusted for individual-level covariates: study condition, visit, sex, baseline age, baseline self-rated health, repeated measured BMI at each visit, baseline educational level; when the outcome was AA-MVPA, models were further adjusted for repeated indicator of rainy conditions at each visit.

As physical activity duration can only take strictly positive values and we could potentially have negative fitted values using Gaussian variance, the assumption of have negative fitted values was checked by plotting residuals versus fitted values, without observing negative fitted values. Antilogarithms of the regression coefficient at Binomial model estimates of these GAMMs represent odds ratios. Also, we evaluated curvilinear relationships of neighborhood walkability attributes with the outcomes at each model visualizing if the residuals contained nonlinear patterns [35].

300 Thin-plate splines smooth terms failed to provide sufficient evidence of a curvilinear relationship in 301 all models, thin-plate splines were replaced by simpler linear terms. Due to the spatial and temporal 302 nature of our data the dependence in the residuals was checked by plotting residuals of the response consequent increase in type I error.

At each model we examined effect modification analysis by study condition (intensive weight-loss lifestyle intervention and unrestricted-energy Mediterranean diet control group). Significance of interactions was assessed by adding cross-product terms between neighborhood walkability and study condition. Then, stratified analyses were performed, by examining the 
association of neighborhood walkability components and AA-MVPA, SRLT-MVPA and SRLT-BW in intervention and control group, separately.

All analyses were conducted in R software version 3.3.3 (R Development Core Team, Vienna, Austria) using 'stats' [36] and 'mgcv' [37] packages and 'corvif' function [38] and ArcGIS V10.5.1

313 software.

\section{3. Results}

\subsection{Descriptive statistics}

Mean age of study participants was 65.0 years (range 55;75) and $48.7 \%$ were women. On average, at baseline, participants did 34.1 minutes MVPA/day based on accelerometer data, 53.1 minutes of SRLT-MVPA/day and 22.0 minutes of brisk walking/day (Table 1). Compared to the control group, more participants in the intervention group engaged in $\geq 150$ minutes/week AA-MVPA and in $\geq 150$ minutes/week SRLT-MVPA at recruitment (Table 1). No other differences between intervention and control group were observed. Participants' walkability index calculated within a $1 \mathrm{Km}$ buffer and its components are provided in Table 1 , mean walkability index was -0.07

323 (range $-2.99 ; 3.25$ ).

324 Table 1. Overall and specific study condition sample characteristics

\begin{tabular}{|c|c|c|c|c|}
\hline & \multicolumn{4}{|c|}{ Study Condition } \\
\hline & All & $\begin{array}{l}\text { Control } \\
\text { group }\end{array}$ & $\begin{array}{l}\text { Intervention } \\
\text { group }\end{array}$ & $p$ \\
\hline Overall & 228 & 122 & 106 & \\
\hline Age (years) & $65.0(4.79)$ & $65.3(4.69)$ & $64.8(4.92)$ & 0.463 \\
\hline Sex & & & & 0.576 \\
\hline Men & $117(51.3 \%)$ & $60(49.2 \%)$ & $57(53.8 \%)$ & \\
\hline Women & $111(48.7 \%)$ & $62(50.8 \%)$ & $49(46.2 \%)$ & \\
\hline $\operatorname{BMI}\left(\mathrm{kg} / \mathrm{m}^{2}\right)$ & $32.7(3.31)$ & $32.5(3.55)$ & $33.0(3.01)$ & 0.226 \\
\hline Educational level & & & & 0.639 \\
\hline Primary school or less & $135(59.2 \%)$ & $70(57.4 \%)$ & $65(61.3 \%)$ & \\
\hline Secondary school or higher & $93(40.8 \%)$ & $52(42.6 \%)$ & $41(38.7 \%)$ & \\
\hline Self-reported health & & & & 0.781 \\
\hline Excellent/very good/good & $156(68.4 \%)$ & $82(67.2 \%)$ & $74(69.8 \%)$ & \\
\hline Fair/poor & $72(31.4 \%)$ & $40(32.5 \%)$ & $32(30.2 \%)$ & \\
\hline Precipitation accelerometer wearing period & & & & 0.827 \\
\hline No rain & $119(52.2 \%)$ & $65(53.3 \%)$ & $54(50.9 \%)$ & \\
\hline Rain & $109(47.8 \%)$ & $57(46.7 \%)$ & $52(49.1 \%)$ & \\
\hline Baseline accelerometer-assessed MVPA (minutes/day) & $34.1(26.2)$ & $32.2(27.5)$ & $36.3(24.7)$ & 0.239 \\
\hline Accelerometer wear time, valid days & $7.82(1.44)$ & $7.80(1.40)$ & $7.85(1.49)$ & 0.779 \\
\hline $\begin{array}{l}\text { Engaging in } \geq 150 \text { minutes/week accelerometer-assessed } \\
\text { MVPA }\end{array}$ & & & & 0.061 \\
\hline No & $89(39.0 \%)$ & $55(45.1 \%)$ & $34(32.1 \%)$ & \\
\hline Yes & $139(61.0 \%)$ & $67(54.9 \%)$ & $72(67.9 \%)$ & \\
\hline Baseline self-reported MVPA (minutes/day) & $53.1(59.5)$ & $49.9(60.7)$ & $56.9(58.1)$ & 0.374 \\
\hline $\begin{array}{l}\text { Engaging in } \geq 150 \text { minutes/week self-reported leisure-time } \\
\text { MVPA }\end{array}$ & & & & 0.026 \\
\hline No & $83(36.4 \%)$ & $53(43.4 \%)$ & $30(28.3 \%)$ & \\
\hline
\end{tabular}




\begin{tabular}{|c|c|c|c|c|}
\hline Yes & $145(63.6 \%)$ & $69(56.6 \%)$ & $76(71.7 \%)$ & \\
\hline Self-reported leisure-time brisk walking (minutes/day) & $22.0(29.6)$ & $20.8(33.2)$ & $23.4(24.9)$ & 0.493 \\
\hline \multicolumn{4}{|l|}{$\begin{array}{l}\text { Engaging in } \geq 45 \text { minutes/day } 6 \text { days/week self-reported } \\
\text { leisure-time brisk walking }\end{array}$} & \multirow[t]{3}{*}{0.124} \\
\hline No & $106(46.5 \%)$ & $63(51.6 \%)$ & $43(40.6 \%)$ & \\
\hline Yes & $122(53.5 \%)$ & $59(48.4 \%)$ & $63(59.4 \%)$ & \\
\hline Walkability index & $-0.07(1.09)$ & $-0.05(1.03)$ & $-0.10(1.16)$ & 0.777 \\
\hline Z-score Residential density & $-0.06(1.02)$ & $-0.03(1.01)$ & $-0.09(1.03)$ & 0.619 \\
\hline Z-score Land use mix & $0.05(1.02)$ & $0.02(1.02)$ & $0.09(1.02)$ & 0.638 \\
\hline Z-score Intersection density & $-0.07(1.02)$ & $-0.05(0.97)$ & $-0.09(1.08)$ & 0.780 \\
\hline Deprivation index & $1.92(0.99)$ & $1.95(1.01)$ & $1.90(0.96)$ & 0.726 \\
\hline \multicolumn{5}{|c|}{$\begin{array}{l}\text { Values shown are } n(\%) \text { for categorical variables and mean (SD) for continuous variables. The } \\
\text { p-values are computed from t-test when row-variable is continuous normal-distributed, } \\
\text { Kruskall-Wallis test when it is continuous non-normal. When row-variable is categorical we used } \\
\text { chi-squared or exact Fisher test when the expected frequencies are less than } 5 \text { in some cell. }\end{array}$} \\
\hline
\end{tabular}

\subsection{Associations of neighborhood walkability components on physical activity duration}

Results of associations between neighborhood walkability and its components in the $1 \mathrm{Km}$ buffer and one-year changes in AA-MVPA, SRLT-MVPA and SRLT-BW are provided in Table 2. When considering the overall sample, AA-MVPA significantly increased per increment in $1 \mathrm{z}$-score neighborhood walkability ( $B=3,44 ; 95 \% C I=0.52 ; 6.36$ minutes per day; $p=0.021$ ). Borderline interaction was detected between study condition (control group and intervention group) and neighborhood walkability $(\mathrm{p}=0.05)$. Stratified analyses showed that each unitary increment in 1 z-score neighborhood walkability was associated with an increase of $6.36(95 \% \mathrm{CI}=2.07 ; 10.64)$ minutes per day among individuals assigned to the intervention group. A non-significant association was observed for the control group.

Intersection density was the component of the walkability index more strongly associated with one-year changes in AA-MVPA $(B=4.32 ; 95 \% \mathrm{CI}=0.19 ; 7.45$ minutes per day; $\mathrm{p}=0.004)$. Although non-significant interaction between study condition and intersection density z-score was detected $(\mathrm{p}$ $=0.11)$, in the stratified analyses an association was observed in the intervention group $(B=6.86 ; 95 \%$ $C I=2.25 ; 11.48 ; p=0.004$ ) but not in the control group. Residential density was not associated with one-year changes in AA-MVPA. Despite the fact that the interaction between study condition and neighborhood walkability was non-significant $(p=0.40)$, stratified analyses showed an association between residential density and one-year changes in AA-MVPA in the intervention group $(B=5.07$; $95 \% \mathrm{CI}=0.11 ; 10.03 ; \mathrm{p}=0.046)$ but not in the control group. In contrast, a borderline inverse association was found for the land use mix component and AA-MVPA $(B=-3.20 ; 95 \% \mathrm{CI}=$ $-6.32 ;-0.09 ; p=0.045)$ in the overall sample, with non-significant associations observed for both groups in the stratified analyses.

Overall, no statistically significant associations were observed between neighborhood walkability or its components and one-year changes in SRLT-MVPA or SRLT-BW (Table 2).

Supplementary Table S1 shows the results of associations between neighborhood walkability and its components in the $0.5 \mathrm{Km}$ buffer and one-year changes in AA-MVPA, SRLT-MVPA, and SRLT-BW. In line with results from walkability measured within the $1 \mathrm{Km}$ buffer, when considering the overall sample, in the $0.5 \mathrm{Km}$ buffer results, increments in neighborhood walkability was associated with an increase in AA-MVPA. Although the interaction between study condition and neighborhood walkability was non-significant $(p=0.08)$, in the stratified analyses an increase in AA-MVPA was only observed in the intervention group. In the $0.5 \mathrm{Km}$ buffer, the association between neighborhood walkability and AA-MVPA seemed to be driven by the intersection density component. In the overall sample, neighborhood walkability and its components, residential 
density, intersection density and land use mix within the $0.5 \mathrm{Km}$ buffer, were not associated with one-year changes in SRLT-MVPA (Table S1). An interaction was detected between study condition and land use mix component $(\mathrm{p}=0.03)$. Land use mix component within the $0.5 \mathrm{Km}$ buffer was associated with a one-year decrease in SRLT-MVPA at the intervention group. 
Table 2. Summary of associations between neighborhood walkability and its components measured in the $1 \mathrm{Km}$ buffer, and accelerometer-assessed moderate-to-vigorous physical activity (AA-MVPA), self-reported leisure-time moderate-to-vigorous physical activity (SRLT-MVPA), and self-reported leisure-time brisk walking (SRLT-BW) in the overall sample $(n=228)$ and after stratification according to the PREDIMED-Plus control $(n=122)$ and intervention $(n=106)$ groups.

\begin{tabular}{|c|c|c|c|c|c|c|c|c|c|}
\hline \multirow{2}{*}{ Predictor Variable } & \multicolumn{3}{|c|}{ Accelerometer-assessed MVPA } & \multicolumn{3}{|c|}{$\begin{array}{c}\text { Self-reported Leisure-Time } \\
\text { MVPA }\end{array}$} & \multicolumn{3}{|c|}{$\begin{array}{c}\text { Self-reported leisure-time brisk } \\
\text { walking }\end{array}$} \\
\hline & B & $95 \% \mathrm{CI}$ & $p$ & B & $95 \% \mathrm{CI}$ & $p$ & B & $95 \% \mathrm{CI}$ & $p$ \\
\hline Walkability index & 3.44 & $0.52 ; 6.36$ & 0.021 & -4.44 & $-10.00 ; 1.13$ & 0.119 & -0.05 & $-2.97 ; 2.87$ & 0.975 \\
\hline$P$ for Interaction & & & 0.055 & & & 0.927 & & & 0.485 \\
\hline Walkability index control group & 0.10 & $-3.94 ; 4.15$ & 0.960 & -4.69 & $-12.80 ; 3.42$ & 0.258 & -0.94 & $-6.08 ; 4.20$ & 0.721 \\
\hline Walkability index intervention group & 6.36 & $2.07 ; 10.64$ & 0.004 & -4.78 & $-12.48 ; 2.91$ & 0.224 & 1.05 & $-2.46 ; 4.55$ & 0.558 \\
\hline Z-score of residential density & 2.92 & $-0.19 ; 6.03$ & 0.067 & -0.16 & $-6.04 ; 5.72$ & 0.958 & -0.41 & $-3.47 ; 2.65$ & 0.794 \\
\hline$P$ for Interaction & & & 0.398 & & & 0.44 & & & 0.311 \\
\hline Z-score of residential density control group & 1.95 & $-2.01 ; 5.91$ & 0.335 & -2.14 & $-10.23 ; 5.93$ & 0.603 & -1.84 & $-6.87 ; 3.19$ & 0.474 \\
\hline Z-score of residential density intervention group & 5.07 & $0.11 ; 10.03$ & 0.046 & 2.76 & $-5.98 ; 11.49$ & 0.537 & 1.66 & $-2.29 ; 5.60$ & 0.412 \\
\hline Z-score intersection density & 4.32 & $1.19 ; 7.45$ & 0.007 & -4.18 & $-10.18 ; 1.82$ & 0.173 & -0.36 & $-3.50 ; 2.79$ & 0.824 \\
\hline$P$ for Interaction & & & 0.111 & & & 0.848 & & & 0.572 \\
\hline Z-score intersection density control group & 1.38 & $-2.98 ; 5.75$ & 0.535 & -4.61 & $-13.38 ; 4.15$ & 0.303 & -1.10 & $-6.67 ; 4.47$ & 0.7 \\
\hline Z-score intersection density intervention group & 6.86 & $2.25 ; 11.48$ & 0.004 & -4.18 & $-12.47 ; 4.12$ & 0.325 & 0.60 & $-3.17 ; 4.37$ & 0.755 \\
\hline Z-score land use mix & -3.20 & $-6.32 ;-0.09$ & 0.045 & -0.77 & $-6.65 ; 5.11$ & 0.798 & 0.70 & $-2.37 ; 3.76$ & 0.656 \\
\hline$P$ for Interaction & & & 0.683 & & & 0.365 & & & 0.421 \\
\hline Z-score land use mix control group & -3.03 & $-7.00 ; 0.94$ & 0.136 & 1.42 & $-6.66 ; 9.50$ & 0.731 & 1.82 & $-3.20 ; 6.84$ & 0.477 \\
\hline Z-score land use mix intervention group & -4.51 & $-9.49 ; 0.47$ & 0.077 & -4.24 & $-12.94 ; 4.46$ & 0.340 & -0.97 & $-4.93 ; 2.98$ & 0.629 \\
\hline
\end{tabular}


Results of associations between neighborhood walkability and its components measured in the $1 \mathrm{Km}$ buffer and dichotomous outcome variables, such as engaging in $\geq 150$ minutes/week of AA-MVPA or SRLT-MVPA and engaging in $\geq 45$ minutes/day 6 days per week of SRLT-BW after one-year, are provided in Table 3. On the whole analytic sample, no statistically significant associations were observed between neighborhood walkability -or its components- and engaging in $\geq 150$ minutes/week AA-MVPA. Significant interactions were detected between study condition and neighborhood walkability $(\mathrm{p}=0.017)$, and between study condition and intersection density $(\mathrm{p}=0.042)$. Stratified analyses found that in those individuals assigned to the intervention group, walkability index $(\mathrm{OR}=1.48 ; 95 \% \mathrm{CI}=1.073 ; 2.054)$ and intersection density $(\mathrm{OR}=1.51 ; 95 \% \mathrm{CI}=$ $1.064 ; 2.145)$ were associated with greater odds of meeting the recommendation of $\geq 150$ minutes/week of AA-MVPA. A non-significant association was observed in those individuals assigned to the intervention group between residential density or land use mix and engaging in $\geq$ 150 minutes/week AA-MVPA. A non-significant association was observed for the control group between neighborhood walkability -or its components- and engaging in $\geq 150$ minutes/week AA-MVPA.

Overall, no statistically significant associations or interactions, were observed between neighborhood walkability or its components and engaging in $\geq 150$ minutes/week of SRLT-MVPA, or in $\geq 45$ minutes/day 6 days per week of SRLT-BW. However, in the stratified analysis, individuals assigned to the control group stowed a significant association between the walkability index and intersection density and lower odds of engaging in $\geq 150$ minutes/week SRLT-MVPA.

Overall no statistically significant associations or interactions were shown between neighborhood walkability or its components in the $0.5 \mathrm{Km}$ buffer and engaging in $\geq 150$ minutes/week of AA-MVPA, SRLT-MVPA or engaging in $\geq 45$ minutes/day 6 days per week of SRLT-BW after one-year (Table S2). 
Table 3. Summary of associations between neighborhood walkability and its components measured in the $1 \mathrm{Km}$ buffer, and engaging in $\geq 150$ minutes/week accelerometer-assessed moderate-to-vigorous physical activity (AA-MVPA), self-reported leisure-time moderate-to-vigorous physical activity (SRLT-MVPA) and engaging in $\geq 45$ minutes/day 6 days per week self-reported leisure-time brisk walking (SRLT-BW) in the overall sample $(n=228)$ and after stratification according the PREDIMED-Plus control $(n=122)$ and intervention $(n=106)$ groups.

\begin{tabular}{|c|c|c|c|c|c|c|c|c|c|}
\hline \multirow[t]{2}{*}{ Predictor Variable } & \multicolumn{3}{|c|}{$\begin{array}{l}\text { Engaging in } \geq 150 \text { minutes/week } \\
\text { accelerometer-assessed MVPA }\end{array}$} & \multicolumn{3}{|c|}{$\begin{array}{l}\text { Engaging in } \geq 150 \text { minutes/week } \\
\text { self-reported leisure-time MVPA }\end{array}$} & \multicolumn{3}{|c|}{$\begin{array}{c}\text { Engaging in } \geq 45 \text { minutes/day } 6 \\
\text { days per week self-reported } \\
\text { leisure-time brisk walking }\end{array}$} \\
\hline & OR & $95 \% \mathrm{CI}$ & $p$ & OR & $95 \% \mathrm{CI}$ & $p$ & OR & $95 \% \mathrm{CI}$ & $P$ \\
\hline Walkability index & 1.06 & $0.81 ; 1.38$ & 0.658 & 0.80 & $0.63 ; 1.02$ & 0.078 & 0.94 & $0.76 ; 1.17$ & 0.569 \\
\hline$P$ for Interaction & & & 0.017 & & & 0.123 & & & 0.107 \\
\hline Walkability index control group & 0.83 & $0.56 ; 1.23$ & 0.358 & 0.67 & $0.47 ; 0.95$ & 0.023 & 0.76 & $0.56 ; 1.04$ & 0.085 \\
\hline Walkability index intervention group & 1.48 & $1.07 ; 2.05$ & 0.018 & 0.98 & $0.68 ; 1.41$ & 0.920 & 1.16 & $0.85 ; 1.59$ & 0.354 \\
\hline Z-score of residential density & 1.02 & $0.78 ; 1.33$ & 0.895 & 0.82 & $0.64 ; 1.06$ & 0.128 & 0.94 & $0.75 ; 1.17$ & 0.57 \\
\hline$P$ for Interaction & & & 0.381 & & & 0.586 & & & 0.269 \\
\hline Z-score of residential density control group & 1.02 & $0.70 ; 1.50$ & 0.905 & 0.78 & $0.56 ; 1.08$ & 0.137 & 0.84 & $0.62 ; 1.14$ & 0.264 \\
\hline Z-score of residential density intervention group & 1.25 & $0.87 ; 1.79$ & 0.224 & 0.89 & $0.59 ; 1.33$ & 0.575 & 1.14 & $0.80 ; 1.64$ & 0.46 \\
\hline Z-score intersection density & 1.12 & $0.84 ; 1.49$ & 0.446 & 0.77 & $0.59 ; 1.01$ & 0.057 & 0.94 & $0.75 ; 1.19$ & 0.619 \\
\hline$P$ for Interaction & & & 0.042 & & & 0.167 & & & 0.166 \\
\hline Z-score intersection density control group & 0.91 & $0.60 ; 1.40$ & 0.682 & 0.64 & $0.44 ; 0.94$ & 0.023 & 0.77 & $0.55 ; 1.07$ & 0.122 \\
\hline Z-scores intersection density intervention group & 1.51 & $1.06 ; 2.14$ & 0.022 & 0.94 & $0.64 ; 1.38$ & 0.763 & 1.15 & $0.82 ; 1.61$ & 0.432 \\
\hline Z-score land use mix & 0.95 & $0.72 ; 1.24$ & 0.687 & 1.21 & $0.95 ; 1.55$ & 0.131 & 1.05 & $0.84 ; 1.32$ & 0.65 \\
\hline$P$ for Interaction & & & 0.71 & & & 0.809 & & & 0.44 \\
\hline Z-score land use mix control group & 0.88 & $0.60 ; 1.29$ & 0.517 & 1.24 & $0.90 ; 1.72$ & 0.187 & 1.14 & $0.84 ; 1.54$ & 0.409 \\
\hline Z-score land use mix intervention group & 0.84 & $0.59 ; 1.20$ & 0.342 & 1.17 & $0.78 ; 1.75$ & 0.447 & 0.91 & $0.64 ; 1.30$ & 0.605 \\
\hline
\end{tabular}


410 intercept participant nested within the neighborhood deprivation index; when the outcome was AA-MVPA, models were further adjusted for repeated indicator of rainy 411 conditions at each visit). 
This study provides new evidence on the association between neighborhood walkability and physical activity in a tailored intervention to increase physical activity in older adults. Among this sub-sample of overweight and obese senior subjects from Palma de Mallorca with metabolic syndrome, living in more favorable walking neighborhood was associated to a significant one-year increase in MVPA, measured with accelerometer. Moreover, the intervention' effects of increasing participants accelerometer-measured MVPA were greater for those living in more walkable neighborhoods.

\subsection{Neighborhood walkability and physical activity}

Urbanization and inactive ageing are transformative trends that have become a global key issue for public health $[7,12]$. Nevertheless, little is known about the effects of the environments in which older adult are encouraged to acquire physical activity behaviors. Our study is the first to examine the potential moderating effects between neighborhood walkability and a physical activity intervention in European older adults, on physical activity.

Tailored intervention programs that target individuals, tend to reach small numbers of people but produce larger effect sizes at short-term [5]. However, urban environments' effects on physical activity can be expected to generally apply to individuals living in the neighborhood, reaching large numbers of people but producing lower effects at the long-term [13].

Over the past two decades, there has been an increase in the number of publications evaluating the effectiveness of physical activity intervention programs among older adults, as well as, exploring the relationship between neighborhood physical environmental attributes and older adults' physical activity. There is evidence of a significant increase of physical activity among older adults during such intervention programs, as summarized in two recent systematic reviews [5, 6]. Separately, two other systematic reviews have summarized the evidence coming mostly from cross sectional studies, indicating strong evidence among older adults for a positive association between neighborhood walkability and leisure-time walking [39] or accelerometer assessed MVPA [40]. Both systematic reviews highlight a need for longitudinal and quasi-experimental designs. Despite the growing amount of literature about neighborhoods' built environments and health, a European systematic review pointed out that only a small proportion of the current evidence refers to European studies; the majority of findings were from only a few cities in North American and Australian regions [41].

The most important contribution of the presented analyses is the methodological approach using a prospective design within a randomized trial. Our findings add to the currently reduced amount of existing evidence determining whether the neighborhood walkability in which older adults live, supports an intervention attempting to increase their physical activity.

We found evidence that the higher walkability index was related to an increase in AA-MVPA during the PREDIMED-Plus physical activity intervention suggesting that living in highly walkable areas supports this type of intervention.

Moreover, our results also highlight the importance of the intersection density and residential density components during a tailored physical activity intervention. Participants living in neighborhoods with more connected streets and with more people living within their neighborhood increased more their AA-MVPA during the physical activity intervention than those living in less connected and dense neighborhoods.

This is likely because higher street connectivity means that the street segment length and block sizes are smaller, and therefore, destinations are more directly accessible in a greater number of alternative travel paths. Additionally, higher residential density provides increased interaction between residents, services users and those on the street, in Jane Jacobs' words, "eyes on the street" [42]. Possibly, higher intersection density and higher residential density areas are supporting features of the neighborhoods during a physical activity intervention and consequently increased older adults' AA-MVPA. 
To date, only one previous study conducted in North America has explored the effects of objective neighborhood walkability on self-reported physical activity among older adults attempting to increase their physical activity levels [43]. Despite the lack of an objective measure of physical activity, King et al found no significant interactions for leisure walking between neighborhood walkability and study arm during a 12-month intervention program [43]. These findings are consistent with our results on SRLT-MVPA. Because SRLT-MVPA encompasses all forms of leisure-time MVPA (brisk walking, walking on trails/hiking, gardening, climbing stairs, and sport activities), we expected weaker associations between this measure and neighborhood walkability. Examining the relationships between neighborhood walkability and SRLT-BW, King and colleagues also found no statistically significant association.

Leisure-time walking encompasses both walking for transportation and walking for recreation. One possible explanation for these findings is that the walkability index [10] used in these studies was primarily designed in relation to travel patterns and not to recreational walking then leisure-time walking, not transport-related, can make this association weaker. King et al found a significant interaction for walking for errands; in other words those living in less walkable neighborhoods significantly decreased their meaningful form of walking, compensating this decrease with other leisure-time activities [43]. Among cross-sectional studies there have been similar findings. Results from the Belgian Environmental Physical Activity Study in Seniors pointed out that neighborhood walkability was not associated with recreational walking; instead it was positively associated with walking for transportation [44]. Studies conducted in North America found that more walkable neighborhoods were associated with transport-related walking as well as overweight status $[45,46]$.

A long-term longitudinal study among an adult population found that during the 12-year follow-up, leisure-time physical activity patterns did not vary by walkability [47]. In line with this finding, we observed that neighborhood walkability was not associated with one-year changes in self-reported leisure time variables (SRLT-MVPA and SRLT-BW). Additionally, other longitudinal results from the Multi-Ethnic Study of Atherosclerosis among middle-aged and older adults, with a median follow-up of 9-years, indicated that walkability features had more influence on changes in transport walking than on changes in leisure walking [48]. These results indicate that neighborhood walkability seems to be more related to transportation behaviors than leisure time ones.

Regarding the accelerometer-assessed outcome, we found that higher baseline values in walkability resulted in increases in AA-MVPA. This positive association seems to be driven by the intersection density component; however, a negative association was found between the land use mix component and AA-MVPA. Previous research has identified the integration of public open spaces into land mix scenarios as having a positive relationship for increased physical activity [24]. However, when considering public open spaces as individual measures, no relationship between access to public open spaces and baseline physical activity was identified with this cohort [49]. Additionally, a recent study showed that among older adults during their time spent within urban green spaces (parks and gardens) sedentary behaviors were more common than physical activities [50]. This indicates that built environments are complex and dynamic systems, and the modelling of the combinations of multiple environmental variables and indices, is needed, as previously highlighted in Sallis et al [51].

\subsection{Strengths and limitations}

The present study has several strengths, including the use of a prospective design within a randomized trial with six-month and one-year follow-up measurements of both self-reported and accelerometer measures of physical activity; while other studies have mostly used cross-sectional data [39, 40] or self-report questionnaires to measure physical activity outcome [43, 52]. Objectively-assessed physical activity through accelerometry, despite not being able to identify domain-specific activities, is considered a more valid assessment of the intensity, duration, and frequency of physical activity than self-reported alternatives, that are also prone to desire biases and over-estimations $[53,54]$. 
Within the area of knowledge of physical activity promotion, interventions that target older adults and those with disabilities are less common [6]. Physical activity intervention programs focused on overweight/obese senior adults reflects an important contribution to a key issue for public health [55-57]. Also, our study was conducted in the context of a European Mediterranean region, adding to the body of evidence that was based on other non-European areas.

There were a number of limitations. Our results should be interpreted with caution since the follow-up period was only one-year, which might be too short to detect major changes. We objectively measured neighborhood walkability using macro-scale features thus, findings using micro-environmental variables (e.g., vegetation, benches, obstacles on sidewalk, type of surface, etc.) and perceived environment measures could supplement our findings [58, 59]. Future studies should explore combinations of environmental features, in order to explain more variation in physical activity than single variables [51]. Study participants in PREDIMED-Plus were not recruited based on geographic characteristics in order to maximize variability in neighborhood walkability, and the PREDIMED-Plus intervention did not focus specifically on the physical environment, as a consequence neighborhood walkability effects on physical activity outcomes may be underestimated. Another study limitation is the lack of a specific measure of active transportation (e.g., walking to a shop), which could have provided additional insights related to the potential impacts of neighborhood walkability on this domain-specific physical activity. Also, as a major common issue of built environments research, we cannot avoid the effects of self-selection. In other words, which came first: the walkable neighbourhood change in residents' behaviours or residents who prefer more active lifestyles choose to live in walkable neighborhoods? Our ability to determine the relationship between cause and effect was confounded by physical activity preference.

Finally, there are other variables that could modify the association between neighborhood walkability and physical activity during a physical activity intervention, such as time participating in the intervention, age, sex, self-rated health, BMI, educational level and neighborhood socioeconomic status; this sub-sample of the ongoing PREDIMED-Plus trial was not powered to perform any of those stratified analyses and other sensitivity analyses. Therefore, our findings should be interpreted with caution and need to be replicated in larger samples, larger follow-up, and from geographically diverse neighborhoods.

\section{5. Conclusions}

This study adds to the growing body of literature on walkable neighborhoods and health. It provides new evidence that it is important to consider such as the neighborhood walkability and built environment when designing and implementing physical activity programs. Results indicate that, among overweight and obese senior adults with metabolic syndrome and assigned to a tailored intervention to increase physical activity, living in a walkable neighborhood appears to be an important factor related to active ageing. This adds to increasing evidence supporting a whole system approach when designing physical activity intervention programs and warrants further investigation.

\section{Supplementary information}

Additional file 1: Table S1. Summary of associations between neighborhood walkability and its components measured in the $0.5 \mathrm{Km}$ buffer and accelerometer-assessed moderate-to-vigorous physical activity (AA-MVPA), self-reported leisure-time moderate-to-vigorous physical activity (SRLT-MVPA) and self-reported leisure-time brisk walking (SRLT-BW). Table S2. Summary of associations between neighborhood walkability and its components measured in the $0.5 \mathrm{Km}$ buffer and engaging in $\geq 150$ minutes/week accelerometer-assessed moderate-to-vigorous physical activity (AA-MVPA), self-reported leisure-time moderate-to-vigorous physical activity (SRLT-MVPA) and engaging in $\geq 45$ minutes/day 6 days per week self-reported leisure-time brisk walking (SRLT-BW).

\section{Abbreviations:}

560 PREDIMED: PREvención con DIeta MEDiterránea; GAMMs: Generalised Additive Mixed Models; CEI-IB: 561 Committee of Research Ethics of the Balearic Islands; HUSE: University Hospital Son Espases; MVPA: 
moderate-to-vigorous physical activity; CVD: cardiovascular disease; RSPAQ: REGICOR Short Physical Activity Questionnaire; AA-MVPA: accelerometer assessed: MVPA; g: gravity units; SRLT-MVPA: self-reported leisure-time moderate-to-vigorous physical activity; SRLT-BW: self-reported leisure-time brisk walking; BMI: body mass index; VIF: variance inflation factor;

\section{Acknowledgments:}

The authors would like to acknowledge the investigators, personnel, and study participants of the PREDIMED-Plus study, as well as all affiliated primary care centers. A.C. thank Dr. Alain F. Zuur and Dra. Elena N. Ieno at Highland Statistics Ltd. for teaching how to deal with the great amount of misery that comes together in the statistical analysis.

\section{Authors' contributions:}

A.C. Conceived the study, conducted the spatial analysis and data preparation for statistical analysis, performed statistical analysis and drafted the manuscript. S.M. Guided the spatial analysis of objective measures of the neighborhood walkability index component' and supervised A.C. in manuscript preparation and reviewed all manuscript drafts. M.R. Assisted with geocoding and data preparation and supervised. J.W. Performed processed accelerometer data, preparation of objective measures of physical activity data for statistical analysis and supervised. J.M. Contributed to the statistical analysis and statistical analysis discussion section of the study. J.K. Conducted the trial and contributed to the statistical analysis. F.J.B-L. Performed processed accelerometer data, preparation of objective measures of physical activity data for statistical analysis and supervised statistical analysis. M.F. principal investigator of the PREDIMED-PLUS trial, member of the steering committee, and reviewed all manuscript drafts. J.S.S. is principal investigator of the PREDIMED-PLUS trial, member of the steering committee, and reviewed all manuscript drafts. D.R. is the principal investigator of the PREDIMED-Plus study in the Balearic Islands and supervised A.C. in study design and manuscript preparation and reviewed all manuscript drafts. All authors read and approved the final manuscript.

\section{Funding:}

586 Instituto de Investigación en Salud Carlos III (PI14/00853, PI16/00662 and PI17/00525), and Consejería de Salud de la Junta de Andalucía (PS0358-2016). Cofounded by FEDER. AstraZeneca Foundation (Young investigator Award on obesity and type 2 diabetes to D.R. 2017). S.M. is supported by an Australian National Health and Medical Research Council fellowship (\#1121035).

\section{Availability of data and materials:}

591 There are restrictions on the availability of data for the PREDIMED-Plus trial, due to the signed consent agreements around data sharing, which only allow access to external researchers for studies following the project purposes. Requestors wishing to access the PREDIMED-Plus trial data used in this study can make a request to the PREDIMED-Plus trial Steering Committee chair: jordi.salas@urv.cat. The request will then be passed to members of the PREDIMED-Plus Steering Committee for deliberation.

\section{Ethics approval and consent to participate:}

597 The Committee of Research Ethics of the Balearic Islands (CEI-IB) approved the study (approval number 598 IB-2242/14-PI). All participants provided written informed consent to participate in the study.

\section{Consent for publication:}

600 Not applicable.

\section{Competing interests:}

602 The authors declare no conflict of interest.

\section{Author details:}

$604{ }^{1}$ Research Group in Cardiovascular Physiopathology and Nutrition Epidemiology (NUTRECOR), Balearic 605 Islands Health Research Institute (IdISBa), University Hospital Son Espases, Palma de Mallorca 07120, Spain; 606 antonicolom@gmail.com (A.C.); jadzia.konieczna@gmail.com (J.K.); mariaadoracion.romaguera@ssib.es (D.R.). 607 2CIBER Fisiopatología de la Obesidad y Nutrición (CIBEROBN), Instituto de Salud Carlos III, Madrid 28029, 608 Spain. ${ }^{3}$ Mary MacKillop Institute for Health Research, Australian Catholic University, Melbourne, Australia. $609{ }^{4}$ Melbourne School of Population and Global Health, University of Melbourne, Melbourne, Australia; 610 suzanne.mavoa@unimelb.edu.au (S.M). ${ }^{5}$ Servicio de SIG y Teledetección,Vicerectorat d'Innovació i 611 Transferència, Universitat de les Illes Balears, Palma de Mallorca 07120, Spain; maurici.ruiz@uib.es. 
${ }^{6}$ Departamento de Enfermería, Facultad de Ciencias de la Salud, Universidad de Málaga - Instituto de 613 Investigación en Biomedicina (IBIMA) Málaga, España; jwarnberg@uma.es (J.W.). ${ }^{7}$ Genomics and 614 Bioinformatics Platform', Balearic Islands Health Research Institute (IdISBa), University Hospital Son Espases; 615 josep.muncunill@ssib.es (J.M.). ${ }^{8}$ Departamento de Salud Pública, Facultad de Medicina, Universidad de Málaga 616 - Instituto de Investigación en Biomedicina (IBIMA) Málaga, España. baron@uma.es (F.J.B-L.). ํUnit of 617 Cardiovascular Risk and Nutrition, Institut Hospital del Mar de Investigaciones Médicas Municipal 618 d'Investigació Mèdica (IMIM), Barcelona, Spain. MFito@imim.es ${ }^{10}$ Rovira i Virgili University, Department of 619 Biochemistry and Biotechnology, Human Nutrition Unit, IISPV, Hospital Universitari Sant Joan de Reus, Reus, 620 Spain. jordi.salas@urv.cat 
624 1. World Health Organization: World Report on Ageing and Health. World Health Organization; 2015:246.

625 2. United Nations, Department of Economic and Social Affairs, Population Division (2017): World Population Ageing 2017. 2017.

3. Guthold R, Stevens GA, Riley LM, Bull FC: Worldwide trends in insufficient physical activity from 2001 to 2016: a pooled analysis of 358 population-based surveys with 1.9 million participants. Lancet Glob Health 2018, 6:e1077-e1086. Lancet Physical Activity Series 2 Executive Committee: Progress in physical activity over the Olympic quadrennium. Lancet 2016, 388:1325-1336.

633 5. Oliveira JS, Sherrington C, Amorim AB, Dario AB, Tiedemann A: What is the effect of health coaching on 634 physical activity participation in people aged 60 years and over? A systematic review of randomised controlled trials. Br J Sports Med 2017, 51:1425-1432. of physical activity interventions for community dwelling older adults: A systematic review of reviews. PLoS ONE 2017, 12:e0180902.

7. World Health Organization: The Global Network for Age-Friendly Cities and Communities: Looking Back Over the Last Decade, Looking Forward to the Next. World Health Organization; 2018.

641 8. Satariano WA, Guralnik JM, Jackson RJ, Marottoli RA, Phelan EA, Prohaska TR: Mobility and aging: new 642 directions for public health action. Am J Public Health 2012, 102:1508-1515.

643 9. Forsyth A, Michael Oakes J, Lee B, Schmitz KH: The built environment, walking, and physical activity: Is 644 the environment more important to some people than others? Transportation Research Part D: Transport and 645 Environment 2009, 14:42-49. 10. Frank LD, Sallis JF, Saelens BE, Leary L, Cain K, Conway TL, Hess PM: The development of a walkability index: application to the Neighborhood Quality of Life Study. Br J Sports Med 2010, 44:924-933.

648 11. Sallis JF, Floyd MF, Rodríguez DA, Saelens BE: Role of built environments in physical activity, obesity, 649 and cardiovascular disease. Circulation 2012, 125:729-737.

650 12. World Health Organization: Global action plan on physical activity 2018-2030: more active people for a 651 healthier world. 2018.

652 13. Sallis JF, Cervero RB, Ascher W, Henderson KA, Kraft MK, Kerr J: An ecological approach to creating 653 active living communities. Annu Rev Public Health 2006, 27:297-322.

654 14. King AC, Sallis JF: Why and how to improve physical activity promotion: lessons from behavioral 655 science and related fields. Prev Med 2009, 49:286-288. 
15. Schröder H, Cárdenas-Fuentes G, Martínez-Gonzalez MA, Corella D, Vioque J, Romaguera D, Alfredo Martinez J, Tinahones FJ, Miranda JL, Estruch R, Bueno-Cavanillas A, Arós F, Marcos A, Tur JA, Wärnberg J, Serra-Majem L, Martín V, Vázquez C, Lapetra J, Pintó X, Vidal J, Daimiel L, Gaforio JJ, Matía-Martín P, Ros E, Castañer O, Lassale C, Ruiz-Canela M, Asensio EM, Basora J, et al.: Effectiveness of the physical activity intervention program in the PREDIMED-Plus study: a randomized controlled trial. Int J Behav Nutr Phys Act 2018, 15:110.

16. Martínez-González MA, Buil-Cosiales P, Corella D, Bulló M, Fitó M, Vioque J, Romaguera D, Martinez JA, Wärnberg J, Lopez-Miranda J, Estruch R, Bueno-Cavanillas A, Arós F, Tur JA, Tinahones F, Serra-Majem L, Martín V, Lapetra J, Vázquez C, Pintó X, Vidal J, Daimiel L, Delgado-Rodríguez M, Matía P, Ros E, Fernández-Aranda F, Botella C, Portillo-Baquedano MP, Lamuela-Raventós RM, Marcos A, et al.: Cohort Profile: Design and methods of the PREDIMED-Plus randomized trial. Int J Epidemiol 2018, 41:377.

17. Alberti KGMM, Eckel RH, Grundy SM, Zimmet PZ, Cleeman JI, Donato KA, Fruchart J-C, James WPT, Loria CM, Smith SC, International Diabetes Federation Task Force on Epidemiology and Prevention, Hational Heart, Lung, and Blood Institute, American Heart Association, World Heart Federation, International Atherosclerosis Society, International Association for the Study of Obesity: Harmonizing the metabolic syndrome: a joint interim statement of the International Diabetes Federation Task Force on Epidemiology and Prevention; National Heart, Lung, and Blood Institute; American Heart Association; World Heart Federation; International Atherosclerosis Society; and International Association for the Study of Obesity. In Circulation. Volume 120. American Heart Association, Inc; 2009:1640-1645.

18. Brownson RC, Hoehner CM, Day K, Forsyth A, Sallis JF: Measuring the built environment for physical activity: state of the science. Am J Prev Med 2009, 36:S99-123.e12.

19. Forsyth A, Van Riper D, Larson N, Wall M, Neumark-Sztainer D: Creating a replicable, valid cross-platform buffering technique: the sausage network buffer for measuring food and physical activity built environments. Int J Health Geogr 2012, 11:14.

680 20. Grasser G, Van Dyck D, Titze S, Stronegger W: Objectively measured walkability and active transport 681 and weight-related outcomes in adults: a systematic review. Int J Public Health 2012, 58:615-625.

682 21. Duncan MJ, Winkler E, Sugiyama T, Cerin E, duToit L, Leslie E, Owen N: Relationships of land use mix with walking for transport: do land uses and geographical scale matter? J Urban Health 2010, 87:782-795. land-use mix measures for use in a national walkability index. Journal of Transport and Land Use 2018, 11.

23. Frank LD, Schmid TL, Sallis JF, Chapman J, Saelens BE: Linking objectively measured physical activity with objectively measured urban form: findings from SMARTRAQ. Am J Prev Med 2005, 28:117-125. 

Adult Population. PLoS ONE 2017, 12:e168148. Q Exerc Sport 2000, 71:S21-9. sedentary behaviour in older adults? Int J Behav Nutr Phys Act 2011, 8:62. output from wrist- and hip-worn monitors. Med Sci Sports Exerc 2014, 46:1816-1824. Whitt-Glover MC, Leon AS: 2011 Compendium of Physical Activities: a second update of codes and MET values. Med Sci Sports Exerc 2011, 43:1575-1581. 2018.

711 35. Zuur AF, Ieno EN: A protocol for conducting and presenting results of regression-type analyses. Methods 712 in Ecology and Evolution 2016, 7:636-645.

713 36. Team RC: R: a Language and Environment for Statistical Computing. 2017.

714 37. Wood S, Scheipl F: Gamm4: Generalized Additive Mixed Models Using Mgcv and Lme4. R Package Version 0.2-3. 7152014.

38. Zuur A, Ieno EN, Walker N, Saveliev AA, Smith GM: Mixed Effects Models and Extensions in Ecology with R. 717 Springer Science \& Business Media; 2009. Activity (CEPA) - Older Adults working group: Built environmental correlates of older adults' total physical activity and walking: a systematic review and meta-analysis. Int J Behav Nutr Phys Act 2017, 14:103. 

Relationship between the physical environment and different domains of physical activity in European adults: a systematic review. BMC Public Health 2012, 12:807.

42. Jacobs JM: The Death and Life of Great American Cities. Penguin Books; 1974.

43. King AC, Salvo D, Banda JA, Ahn DK, Chapman JE, Gill TM, Fielding RA, Demons J, Tudor-Locke C, Rosso A, Pahor M, Frank LD: Preserving older adults' routine outdoor activities in contrasting neighborhood environments through a physical activity intervention. Prev Med 2017, 96:87-93.

44. Van Holle V, Van Cauwenberg J, Van Dyck D, Deforche B, Van de Weghe N, De Bourdeaudhuij I: Relationship between neighborhood walkability and older adults' physical activity: results from the Belgian Environmental Physical Activity Study in Seniors (BEPAS Seniors). Int J Behav Nutr Phys Act 2014, 11:110.

45. Frank L, Kerr J, Rosenberg D, King A: Healthy aging and where you live: community design relationships with physical activity and body weight in older Americans. J Phys Act Health 2010, 7 Suppl 1:S82-90.

46. King AC, Sallis JF, Frank LD, Saelens BE, Cain K, Conway TL, Chapman JE, Ahn DK, Kerr J: Aging in neighborhoods differing in walkability and income: associations with physical activity and obesity in older adults. Soc Sci Med 2011, 73:1525-1533.

47. Creatore MI, Glazier RH, Moineddin R, Fazli GS, Johns A, Gozdyra P, Matheson FI, Kaufman-Shriqui V, Rosella LC, Manuel DG, Booth GL: Association of Neighborhood Walkability With Change in Overweight, Obesity, and Diabetes. JAMA 2016, 315:2211-2220.

48. Hirsch JA, Moore KA, Clarke PJ, Rodríguez DA, Evenson KR, Brines SJ, Zagorski MA, Diez-Roux AV: Changes in the built environment and changes in the amount of walking over time: longitudinal results from the multi-ethnic study of atherosclerosis. Am J Epidemiol 2014, 180:799-809.

49. Colom A, Fiol M, Ruiz M, Compa M, Morey M, Moñino M, Romaguera D: Association between Access to Public Open Spaces and Physical Activity in a Mediterranean Population at High Cardiovascular Risk. Int J Environ Res Public Health 2018, 15:1285.

50. Miralles-Guasch C, Dopico J, Delclòs-Alió X, Knobel P, Marquet O, Maneja-Zaragoza R, Schipperijn J, Vich G: Natural Landscape, Infrastructure, and Health: The Physical Activity Implications of Urban Green Space Composition among the Elderly. Int J Environ Res Public Health 2019, 16:3986.

51. Sallis JF, Cerin E, Conway TL, Adams MA, Frank LD, Pratt M, Salvo D, Schipperijn J, Smith G, Cain KL, Davey R, Kerr J, Lai P-C, Mitáš J, Reis R, Sarmiento OL, Schofield G, Troelsen J, Van Dyck D, De Bourdeaudhuij I, Owen N: Physical activity in relation to urban environments in 14 cities worldwide: a cross-sectional study. Lancet 2016, 387:2207-2217.

52. Kerr J, Norman GJ, Adams MA, Ryan S, Frank L, Sallis JF, Calfas KJ, Patrick K: Do neighborhood environments moderate the effect of physical activity lifestyle interventions in adults? Health Place 2010, 
760 53. Shephard RJ: Limits to the measurement of habitual physical activity by questionnaires. Br J Sports Med

761 2003, 37:197-206- discussion 206.

762 54. Prince SA, Adamo KB, Hamel ME, Hardt J, Connor Gorber S, Tremblay M: A comparison of direct versus 763 self-report measures for assessing physical activity in adults: a systematic review. Int J Behav Nutr Phys Act $764 \quad 2008,5: 56$.

765 55. Lee I-M, Shiroma EJ, Lobelo F, Puska P, Blair SN, Katzmarzyk PT, Lancet Physical Activity Series Working 766 Group: Effect of physical inactivity on major non-communicable diseases worldwide: an analysis of burden 767 of disease and life expectancy. Lancet 2012, 380:219-229.

768 56. Ekelund U, Steene-Johannessen J, Brown WJ, Fagerland MW, Owen N, Powell KE, Bauman A, Lee I-M, 769 Lancet Physical Activity Series 2 Executive Committe, Lancet Sedentary Behaviour Working Group: Does 770 physical activity attenuate, or even eliminate, the detrimental association of sitting time with mortality? A 771 harmonised meta-analysis of data from more than 1 million men and women. Lancet 2016, 388:1302-1310.

772 57. Ding D, Lawson KD, Kolbe-Alexander TL, Finkelstein EA, Katzmarzyk PT, van Mechelen W, Pratt M, 773 Lancet Physical Activity Series 2 Executive Committee: The economic burden of physical inactivity: a global 774 analysis of major non-communicable diseases. Lancet 2016, 388:1311-1324.

775 58. Kweon B-S, Ellis CD, Lee S-W, Rogers GO: Large-Scale Environmental Knowledge: Investigating the 776 Relationship Between Self-Reported and Objectively Measured Physical Environments. Environment and 777 Behavior 2016, 38:72-91.

778 59. Van Cauwenberg J, De Bourdeaudhuij I, Clarys P, Nasar J, Salmon J, Goubert L, Deforche B: Street 779 characteristics preferred for transportation walking among older adults: a choice-based conjoint analysis 780 with manipulated photographs. Int J Behav Nutr Phys Act 2016, 13:6-17. 
Figures

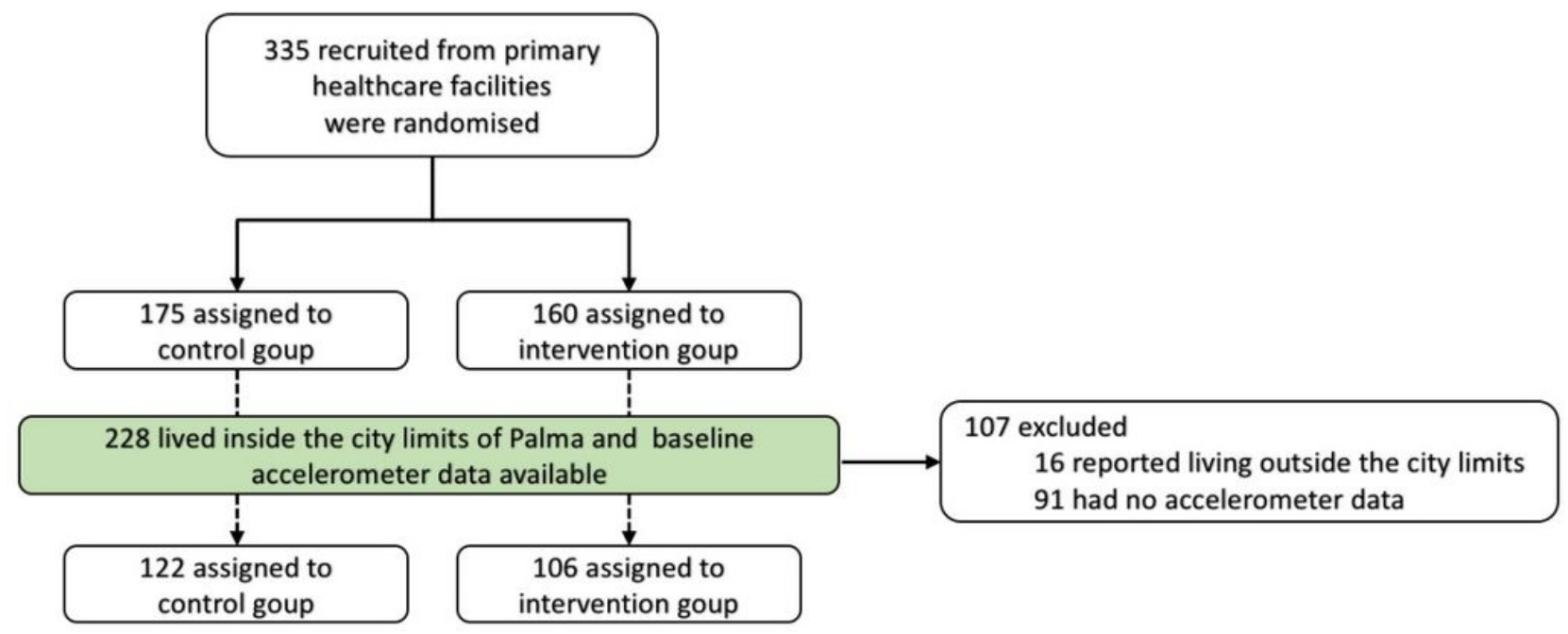

\section{Figure 1}

Flow chart in this analysis of the PREDIMED-Plus trial. 